\title{
HighER-ORDER CHANGE OF THE HAMILTONIAN (ENERGY) FOR NEARLY HOMOCLINIC ORBITS
}

\author{
Richard Haberman and Eric K. Ho \\ Dedicated to the memory of Charles Lange.
}

\begin{abstract}
Conservative dynamical systems with a homoclinic orbit are analyzed under a small dissipative perturbation. The asymptotic expansions of a nearly homoclinic orbit are shown to fail as the surrounding saddle regions are approached. A weakly nonlinear analysis of the surrounding saddle regions is derived assuming the dissipation can be approximated by linear damping in the neighborhood of the saddle point. By matching (to sufficiently high order) the asymptotic expansion of the nearly homoclinic orbit to the solutions in the surrounding saddle regions, the change in the Hamiltonian (the energy dissipation) over a complete nearly homoclinic orbit is obtained. We show there is a new higher-order logarithmic correction to the well-known Melnikov integral for the change of the Hamiltonian from one saddle approach to the next. Numerical computations of this change in the Hamiltonian for a double-well potential are shown to compare favorably with the asymptotic result.
\end{abstract}

\section{Introduction}

We analyze Hamiltonian systems with double homoclinic orbits connecting a saddle point to itself and surrounding two competing centers. Examples include double-well potentials and the strongly nonlinear Hamiltonian system describing generic weakly nonlinear 1-1 primary resonance [see, for example, Guckenheimer and Holmes (1983) and Kevorkian and Cole (1981)]. Other examples are described by Neishtadt (1991) and Henrard (1993). With a small dissipative perturbation, the method of averaging is used to describe the long time behavior of weakly damped strongly nonlinear oscillations. The method of averaging fails as the solution approaches the unperturbed homoclinic orbit. Timofeev (1978) showed that the slow passage through an unperturbed separatrix (homoclinic orbit) involves a large sequence of nearly homoclinic orbits. This was generalized by Neishtadt (1986) and Tennyson et al. (1986). Henrard (1993) summarized, justified, and improved various estimates and illustrated many significant applications. Bourland and Haberman $(1990,1994)$ showed that the nonlinear oscillations obtained by averaging and the nearly homoclinic orbits could be connected by the method of matched asymptotic expansions.

The sequence of nearly homoclinic orbits involves a mapping from one saddle approach to the next. It is now well-known (and presumably known by Poincaré) that the change in the energy over one complete nearly homoclinic orbit can be approximated by what we call the Melnikov integral [see, for example, Guckenheimer and

Received April 25, 1994, revised September 19, 1994.

1991 Mathematics Subject Classification: 34E05, 34E10, 58F30, 34C35, 34C15, $70 \mathrm{~K} 05$.

Key words and phrases: homoclinic orbits, saddle points, Hamiltonian systems, dissipation, perturbations, matched asymptotic expansions, nonlinear differential equations. 
Holmes (1983) and Wiggins (1990)]. The boundaries of the basins of attraction are the stable manifolds of the saddle point. The boundaries of the basin of attraction are tightly intertwined, spiral-like, and near the unperturbed homoclinic orbit consist of a large sequence of solitary pulses in which Melnikov-type energy estimates apply. Bourland and Haberman (1991) obtained accurate asymptotic formulas for the boundaries of the basins of attraction for double-well potentials, and a similar result was obtained by Haberman and Ho (1995a) for the dissipatively perturbed Hamiltonian system corresponding to primary resonance. The Melnikov integral for the change in the energy along a nearly homoclinic orbit is also very important in the calculation of the probability of capture into either stable attractor as discussed by Neishtadt (1991).

The usual Melnikov integral is a leading-order approximation to the exact change of the energy. If the dissipative perturbation to the Hamiltonian system is $O(\varepsilon)$, then the leading-order change of the energy over one nearly homoclinic orbit is $O(\varepsilon)$. Here, we assume the Hamiltonian at each saddle approach is $O(\varepsilon)$ since this corresponds to nearly homoclinic orbits. In the present paper, we analyze conservative systems with a homoclinic orbit and derive a more accurate expression for the change of the energy (the change $\Delta H$ of the Hamiltonian) from one saddle approach to the next along a nearly homoclinic orbit:

$$
\Delta H \sim-\varepsilon M+\varepsilon^{2} \ln \varepsilon\left(\frac{\beta}{k}\right)\left(\frac{H_{+}+H_{-}}{2 \varepsilon}\right)+\cdots
$$

where $M$ is the usual Melnikov integral, $\left(H_{+}+H_{-}\right) / 2$ is the average of the leadingorder energies at the saddle regions which surround this nearly homoclinic orbit, $\beta$ is the linear damping coefficient of the perturbation at the saddle point of the unperturbed system, and $\mathrm{k}$ is the exponential growth and decay rate at the saddle point. Thus, this new dissipation is due to linear damping near the saddle point. In (1.1), $\left(H_{+}+H_{-}\right) / 2 \varepsilon=O(1)$ since the Hamiltonians are $O(\varepsilon)$.

For double-well potentials, the probability of capture into the left and right wells is determined from (1.1), and Haberman and Ho (1995b) show that the probability of capture is not affected by this $O\left(\varepsilon^{2} \ln \varepsilon\right)$ energy correction. For general perturbed Hamiltonian systems, Ho (1993) shows that (1.1) is still valid (with appropriate modifications for the definitions of $\beta$ and $k$ ). However, Haberman and Ho (1995b) show that the probability of capture is changed by $O(\varepsilon \ln \varepsilon)$ from the usual Melnikov probabilities when the topology of the double homoclinic orbit is twisted as in the Hamiltonian system associated with primary resonance.

When the trajectories approach the unperturbed homoclinic orbit (separatrix), the solution behaves differently in different parts of the trajectory. The region away from the saddle point, which we call the nearly homoclinic orbit region, is analyzed in Section 3 with sufficiently high asymptotic accuracy. In Section 4, we do a weakly nonlinear analysis of the neighborhood of the saddle point. This is valid in the saddle regions which surround the nearly homoclinic region. The asymptotic expansion for the nearly homoclinic orbit is matched to the asymptotic expansions of the solution in the surrounding saddle regions. In this way, the change of the energy (1.1) between two saddle approaches is derived in Section 5. The change in the Hamiltonian (energy) between successive saddle approaches is computed numerically in Section 6 for a specific double-well potential and is favorable compared with the asymptotic formula (1.1) including the new $O\left(\varepsilon^{2} \ln \varepsilon\right)$ term. 


\section{Conservative systems with a homoclinic orbit}

We consider conservative systems with a homoclinic orbit under a small dissipative perturbation:

$$
\frac{d^{2} y}{d t^{2}}+V_{y}(y)=-\varepsilon h\left(y, \frac{d y}{d t}\right),
$$

where $V$ is the potential. We use the corresponding force $f(y)\left(f(y)=-V_{y}(y)\right.$ or $\left.V=-\int f(y) d y\right)$. The energy of this system is $H=\frac{1}{2}\left(\frac{d y}{d t}\right)^{2}-\int f(y) d y$. A conservative system with a center and a saddle point and a conservative system with a double-well potential are examples of unperturbed systems $(\varepsilon=0)$ with a homoclinic orbit, whose phase portraits are sketched in Figure 1. With a dissipative perturbation, there are nearly homoclinic orbits, as illustrated in Figure 2. Figure 3 shows a typical timedependent nearly homoclinic orbit and the surrounding saddle regions.

We assume the perturbation $h\left(y, \frac{d y}{d t}\right)$ is purely dissipative, by which we mean that $h\left(y, \frac{d y}{d t}\right)$ is an odd function of $\frac{d y}{d t}$. Furthermore, we assume $h\left(y, \frac{d y}{d t}\right)$ is or can be approximated (using Taylor series) by linear damping, $\beta \frac{d y}{d t}$, in the neighborhood of the saddle point. Later, we show that this assumption of linearity of the damping in the neighborhood of the saddle point is essential to our calculation of the $O\left(\varepsilon^{2} \ln \varepsilon\right)$ correction to the energy dissipation.
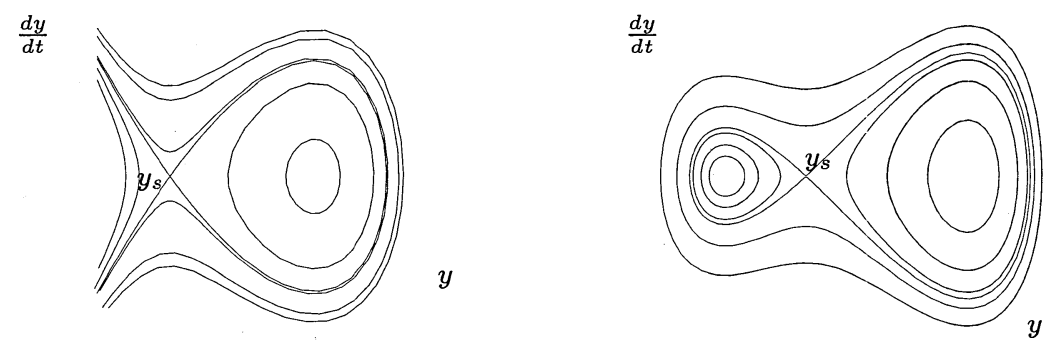

FIGURE 1. Conservative systems with homoclinic orbit $(\varepsilon=0)$

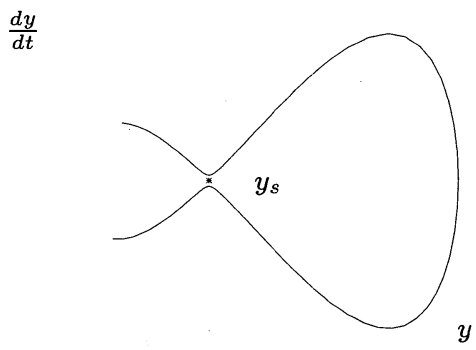

Figure 2. Phase portrait of nearly homoclinic orbit 


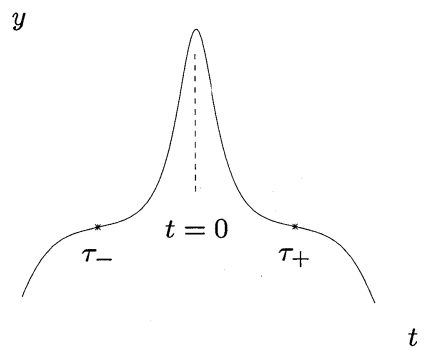

Figure 3. Time-dependent nearly homoclinic orbit

\section{Nearly homoclinic orbit}

In this section, we consider a nearly homoclinic orbit. Here, the solution of the unperturbed system $(\varepsilon=0)$ is assumed to be a homoclinic solution, a single solitary pulse denoted by $y_{0}(t)$. The solution of the perturbed system $(\varepsilon \neq 0)$ which we call a nearly homoclinic orbit can be assumed to have the following expansion

$$
y=y_{0}+\varepsilon y_{1}+\varepsilon^{2} y_{2}+\cdots \text {. }
$$

Since $y$ is near $y_{0}, f(y) \sim f\left(y_{0}\right)+\left(y-y_{0}\right) f_{y}\left(y_{0}\right)+\frac{1}{2}\left(y-y_{0}\right)^{2} f_{y y}\left(y_{0}\right)+\frac{1}{6}\left(y-y_{0}\right)^{3} f_{y y y}\left(y_{0}\right)+$ $\cdots$, and the system (2.1) becomes:

$$
\begin{aligned}
\frac{d^{2} y_{0}}{d t^{2}}+ & \varepsilon \frac{d^{2} y_{1}}{d t^{2}}+\varepsilon^{2} \frac{d^{2} y_{2}}{d t^{2}}+\cdots \\
= & f\left(y_{0}\right)+\left[\varepsilon y_{1}+\varepsilon^{2} y_{2}+\cdots\right] f_{y}\left(y_{0}\right)+\frac{1}{2}\left(\varepsilon y_{1}+\cdots\right)^{2} f_{y y}\left(y_{0}\right) \\
& -\varepsilon h\left(y_{0}, y_{0 t}\right)-\varepsilon^{2}\left[h_{y}\left(y_{0}, y_{0 t}\right) y_{1}+y_{1_{t}} h_{v}\left(y_{0}, y_{0 t}\right)\right]+\cdots
\end{aligned}
$$

where $h_{y}=\frac{\partial}{\partial y} h\left(y, \frac{d y}{d t}\right)$ and $h_{v}=\frac{\partial}{\partial\left(\frac{d y}{d t}\right)} h\left(y, \frac{d y}{d t}\right)$.

To leading order in (3.2):

$$
\frac{d^{2} y_{0}}{d t^{2}}=f\left(y_{0}\right)
$$

We assume the leading-order term $y_{0}(t)$ is a homoclinic orbit, approaching the saddle point $y_{0} \rightarrow y_{s}$ as $t \rightarrow \pm \infty$ where $y_{s}$ is defined by $f\left(y_{s}\right)=0$. In addition, we assume that the center of this homoclinic orbit occurs at $t=0$, so that the solution $y_{0}(t)$ is an even function of $t$.

To determine part of the asymptotic expansions of the homoclinic orbit, we approximate its defining differential equation (3.3) in the neighborhood of the saddle point:

$$
\frac{d^{2}\left(y_{0}-y_{s}\right)}{d t^{2}}=f_{y}\left(y_{s}\right)\left(y_{0}-y_{s}\right)+\frac{1}{2} f_{y y}\left(y_{s}\right)\left(y_{0}-y_{s}\right)^{2}+\cdots
$$

As the solution approaches the saddle regions, i.e., as $t \rightarrow \pm \infty$, we insist that there is no exponentially growing term. Consequently, by solving (3.4), $y_{0}$ has asymptotic 
expansions:

$$
\begin{aligned}
& y_{0} \sim y_{s}+\alpha_{0} e^{k t}+\frac{\alpha_{0}^{2} f_{y y}\left(y_{s}\right)}{6 k^{2}} e^{2 k t}+\cdots, \quad \text { as } t \rightarrow-\infty \\
& y_{0} \sim y_{s}+\alpha_{0} e^{-k t}+\frac{\alpha_{0}^{2} f_{y y}\left(y_{s}\right)}{6 k^{2}} e^{-2 k t}+\cdots, \quad \text { as } t \rightarrow \infty,
\end{aligned}
$$

where $k=\sqrt{f_{y}\left(y_{s}\right)}$ is the exponential growth and decay rate corresponding to the linear analysis around the saddle point (see (3.4)). The asymptotic formula (3.5a) is valid for the saddle point preceding the homoclinic orbit, while (3.5b) is for the following saddle point. The symmetry of $y_{0}$ around $t=0$ has been used to obtain (3.5b) from (3.5a). Since the homoclinic orbit is unique, $\alpha_{0}$ is a known constant which can be determined for a given potential from the integral representation of a homoclinic orbit (but we do not need its value here).

Higher-order terms satisfy the differential equation

$$
\frac{d^{2} y_{i}}{d t^{2}}-f_{y}\left(y_{0}\right) y_{i}=R_{i}, \quad i=1,2,3, \ldots
$$

where $R_{i}$ can be determined from (3.2) and is a function of $y_{j}$ and $y_{j_{t}}$ where $j<i$. In order to solve for $y_{i}$, we use the method of variation of parameters, which requires two homogeneous solutions, one being $y_{0}$. Since $y_{0 t}$ is a nice function, its asymptotic expansion can be determined by differentiating (3.5b) term-by-term:

$$
y_{0 t} \sim-k \alpha_{0} e^{-k t}-\frac{\alpha_{0}^{2} f_{y y}\left(y_{s}\right)}{3 k} e^{-2 k t}+\cdots, \quad \text { as } t \rightarrow \infty .
$$

Since $y_{0}$ is even around $t=0, y_{0}$ is odd around $t=0$. The second homogeneous solution $y_{H}$ can be chosen to be an even function of $\mathrm{t}$ since the linear differential operator in (3.6) has no term with the first derivative and $f_{y}\left(y_{0}\right)$ must be an even function of $t$. This second homogeneous solution can be obtained by considering the Wronskian relationship between $y_{0}$ and $y_{H}$ :

$$
y_{0} y_{H_{t}}-y_{0 t} y_{H}=1 \text {. }
$$

We will only need to notice that $y_{H}$ is even around $t=0$ and has the following important asymptotic expansion (derived in the Appendix),

$$
y_{H} \sim \frac{-1}{2 \alpha_{0} k^{2}}\left[e^{k t}-\frac{\alpha_{0} f_{y y}\left(y_{s}\right)}{k^{2}}-\frac{\alpha_{0}^{2}}{4 k} \sigma t e^{-k t}\right]+\ldots, \quad \text { as } t \rightarrow \infty,
$$

where the parameter

$$
\sigma=f_{y y y}\left(y_{s}\right)-\frac{5 f_{y y}\left(y_{s}\right)}{3 k^{2}} f_{y y}\left(y_{s}\right)
$$

represents the effect on the system of the nonlinearity of the potential (quadratic and cubic nonlinearities of the force) in the neighborhood of the saddle point. The proper mathematical analysis here and in Section 4 requires the inclusion of the $t e^{-k t}$ term.

The first correction $y_{1}$ to the homoclinic orbit due to the purely dissipative perturbation satisfies the following equation:

$$
\frac{d^{2} y_{1}}{d t^{2}}-f_{y}\left(y_{0}\right) y_{1}=-h\left(y_{0}, \frac{d y_{0}}{d t}\right)
$$


By the method of variation of parameters, $y_{1}$ can be represented by

$$
y_{1}=\left[\beta_{1}-\int_{0}^{t} h\left(y_{0}, y_{0 \hat{t}}\right) y_{0 \hat{t}} d \hat{t}\right] y_{H}+\left[\alpha_{1}+\int_{0}^{t} h\left(y_{0}, y_{0 \hat{t}}\right) y_{H} d \hat{t}\right] y_{0 t},
$$

where $\alpha_{1}$ and $\beta_{1}$ are arbitrary constants (multiples of the homogeneous solutions). Since $y_{0}$ is even, the variable coefficient in the linear differential equation (3.11) is even around $t=0$. Because $\mathrm{h}$ is odd around $t=0$, there exists a particular solution of (3.11) which is odd around $t=0$. The solution of (3.11) given by (3.12) is odd around $t=0$, except for the homogeneous solution $\beta_{1} y_{H}$ which is even.

To approximate $y_{1}$ asymptotically in the neighborhood of the saddle point, we note that by our earlier assumption $h\left(y_{0}, y_{0 t}\right)$ can be approximated by $\beta \frac{d y_{0}}{d t}$, and hence $h\left(y_{0}, y_{0}\right)-\beta \frac{d y_{0}}{d t}=O\left(e^{-2 k t}\right)$. To approximate the following term in (3.12) asymptotically as the saddle point is approached, we use the asymptotic behavior of $y_{0}(3.6), y_{H}(3.9)$, and $h\left(y, \frac{d y}{d t}\right)$ :

$$
\begin{aligned}
y_{0 t} \int_{0}^{t} h\left(y_{0}, y_{0 \hat{t}}\right) y_{H} d \hat{t}= & y_{0_{t}} \int_{0}^{t} \beta y_{0 \hat{t}} y_{H} d \hat{t}+y_{0 t} \int_{0}^{t}\left\{\left[h\left(y_{0}, y_{0 \hat{t}}\right)-\beta y_{0 \hat{t}}\right] y_{H}\right\} d \hat{t} \\
& \sim-\frac{\beta \alpha_{0}}{2} t e^{-k t}+O\left(e^{-k t}\right), \quad \text { as } t \rightarrow \infty
\end{aligned}
$$

Later we will show that $t e^{-k t}$ will generate $O\left(\varepsilon^{\frac{3}{2}} \ln \varepsilon\right)$ terms in the saddle region because the saddle time variable will be shown to be just a logarithmic shift of the homoclinic time variable. These terms are very important and essential to our calculation of $O\left(\varepsilon^{2} \ln \varepsilon\right)$ terms for the change of the energy. By (3.12), (3.13), and the asymptotic expansion of $y_{H}(3.9), y_{1}$ has an asymptotic expansion as the subsequent saddle point is approached:

$$
y_{1}=\frac{-Q}{2 k^{2} \alpha_{0}}\left[e^{k t}-\frac{\alpha_{0} f_{y y}\left(y_{s}\right)}{k^{2}}\right]-\left(\frac{\alpha_{0} \beta}{2}-\frac{\alpha_{0} Q \sigma}{8 k^{3}}\right) t e^{-k t}+O\left(e^{-k t}\right), \text { as } t \rightarrow \infty
$$

where we have introduced $Q$ as follows

$$
Q=\beta_{1}-\int_{0}^{\infty} h\left(y_{0}, y_{0 t}\right) y_{0 t} d t
$$

Later, we will show that $\varepsilon Q$ is the leading-order energy at the subsequent saddle approach. We also introduce the Melnikov integral $M$,

$$
M=2 \int_{0}^{\infty} h\left(y_{0}, y_{0}\right) y_{0 t} d t
$$

which represents the well-known leading-order change of the energy over a homoclinic orbit. From (3.15) and (3.16), we have

$$
Q=\beta_{1}-\frac{1}{2} M
$$

Similarly, $y_{1}$ has an asymptotic expansion as the preceding saddle point is approached. Because of the homogeneous solution, $y_{1}$ has different asymptotic expansions as $t \rightarrow-\infty$ and $t \rightarrow+\infty$. From (3.12) as $t \rightarrow-\infty$, the term $\beta_{1}-\frac{1}{2} M$ is replaced 
by $\beta_{1}+\frac{1}{2} M=Q+M$. Thus,

$$
\begin{aligned}
y_{1}= & \frac{-(Q+M)}{2 k^{2} \alpha_{0}}\left[e^{-k t}-\frac{\alpha_{0} f_{y y}\left(y_{s}\right)}{k^{2}}\right] \\
& -\left(\frac{\alpha_{0} \beta}{2}-\frac{\alpha_{0}(Q+M) \sigma}{8 k^{3}}\right) t e^{k t}+O\left(e^{k t}\right), \quad \text { as } t \rightarrow-\infty .
\end{aligned}
$$

For the next-order term in the nearly homoclinic solution $y_{2}$, the $O\left(\varepsilon^{2}\right)$ terms of (3.2) must be considered:

$$
\frac{d^{2} y_{2}}{d t^{2}}-f_{y}\left(y_{0}\right) y_{2}=R_{2}=-y_{1} h_{y}\left(y_{0}, y_{0 t}\right)-y_{1 t} h_{v}\left(y_{0}, y_{0 t}\right)+\frac{1}{2} y_{1}^{2} f_{y y}\left(y_{0}\right)
$$

By the method of variation of parameters, $y_{2}$ can be represented by

$$
y_{2}=\left(\beta_{2}+\int_{0}^{t} R_{2}(\hat{t}) y_{0 t} d \hat{t}\right) y_{H}+\left(\alpha_{2}-\int_{0}^{t} R_{2}(\hat{t}) y_{H} d \hat{t}\right) y_{0 t}
$$

where $\alpha_{2}$ and $\beta_{2}$ are arbitrary constants multiplying the homogeneous solutions. Parts of $y_{2}$ are even and parts are odd, and thus $y_{2}$ has different asymptotic expansions as $t \rightarrow-\infty$ and $t \rightarrow+\infty$. By the method of undetermined coefficients and the asymptotic behavior of $y_{0}(3.5)$ and $y_{1}(3.14)$ and (3.18), we obtain the asymptotic expansions of $y_{2}$ as the surrounding saddles are approached:

$$
\begin{aligned}
& y_{2} \sim \frac{Q^{2} f_{y y}\left(y_{s}\right)}{24 \alpha_{0}^{2} k^{6}} e^{2 k t}+\left(\frac{\beta Q}{4 k^{2} \alpha_{0}}+\frac{Q^{2} \sigma}{16 k^{5} \alpha_{0}}\right) t e^{k t}+\cdots \quad \text { as } t \rightarrow \infty, \\
& y_{2} \sim \frac{(Q+M)^{2} f_{y y}\left(y_{s}\right)}{24 \alpha_{0}^{2} k^{6}} e^{-2 k t}+\left(\frac{\beta(Q+M)}{4 k^{2} \alpha_{0}}+\frac{(Q+M)^{2} \sigma}{16 k^{5} \alpha_{0}}\right) t e^{-k t}+\cdots \\
& \text { as } t \rightarrow-\infty
\end{aligned}
$$

In conclusion, as the solution approaches the subsequent saddle point, i.e., as $t \rightarrow \infty$, the nearly homoclinic orbit has the asymptotic expansion:

$$
\begin{aligned}
y \sim & y_{s}+\alpha_{0} e^{-k t}+\frac{\alpha_{0}^{2} f_{y y}\left(y_{s}\right)}{6 k^{2}} e^{-2 k t} \\
& -\varepsilon\left\{\frac{Q}{2 k^{2} \alpha_{0}}\left[e^{k t}-\frac{\alpha_{0} f_{y y}\left(y_{s}\right)}{k^{2}}\right]+\left(\frac{\beta \alpha_{0}}{2}-\frac{\alpha_{0} Q \sigma}{8 k^{3}}\right) t e^{-k t}\right\} \\
& +\varepsilon^{2}\left[\frac{Q^{2}}{24 \alpha_{0}^{2} k^{6}} f_{y y}\left(y_{s}\right) e^{2 k t}+\left(\frac{\beta Q}{4 k^{2} \alpha_{0}}+\frac{Q^{2} \sigma}{16 k^{5} \alpha_{0}}\right) t e^{k t}+\cdots\right] \\
& +\cdots \quad \text { as } t \rightarrow \infty
\end{aligned}
$$

As the solution approaches the prior saddle region, the nearly homoclinic orbit has 
the following asymptotic expansion:

$$
\begin{aligned}
y \sim & y_{s}+\alpha_{0} e^{k t}+\frac{\alpha_{0}^{2} f_{y y}\left(y_{s}\right)}{6 k^{2}} e^{2 k t} \\
& -\varepsilon\left\{\frac{Q+M}{2 k^{2} \alpha_{0}}\left[e^{-k t}-\frac{\alpha_{0} f_{y y}\left(y_{s}\right)}{k^{2}}\right]+\left(\frac{\beta \alpha_{0}}{2}-\frac{\alpha_{0}(Q+M) \sigma}{8 k^{3}}\right) t e^{k t}\right\} \\
& +\varepsilon^{2}\left[\frac{(Q+M)^{2}}{24 \alpha_{0}^{2} k^{6}} f_{y y}\left(y_{s}\right) e^{-2 k t}+\left(\frac{\beta(Q+M)}{4 k^{2} \alpha_{0}}+\frac{(Q+M)^{2} \sigma}{16 k^{5} \alpha_{0}}\right) t e^{-k t}+\cdots\right] \\
& +\cdots \quad \text { as } t \rightarrow-\infty .
\end{aligned}
$$

We use these asymptotic expansions as the outer (nearly homoclinic orbit) solution in the matching process between the nearly homoclinic orbit region and the surrounding saddle regions.

\section{Solution in successive saddle regions}

For the nearly homoclinic orbit, the solution spends a long time in the neighborhood of the surrounding saddle points. As shown in the last section, the asymptotic behavior can be represented by (3.21). The leading-order term of the asymptotic expansion of the nearly homoclinic orbit (outer) exponentially decays to $y_{s}$ as $t \rightarrow \pm \infty$. From (3.21a) and (3.21b), the $O(\varepsilon)$ term exponentially grows as $t \rightarrow \pm \infty$. Thus, the expansions fail as $t \rightarrow \pm \infty$. In this section, we show that different asymptotic expansions are necessary in the neighborhood of the surrounding saddle points. We determine the differential equations valid in the surrounding saddle regions by rescaling the solutions based on (3.21). This is the fundamental idea behind the method of matched asymptotic expansions.

First, we consider the failure as $t \rightarrow+\infty$. The correct scale is derived by assuming $e^{-k t}=O\left(\varepsilon e^{k t}\right)$ as $t \rightarrow \infty$. This type of failure of an asymptotic expansion corresponds to shifting the time variable [Lange (1983)] by a logarithmically large amount, $-\frac{1}{2 k} \ln \varepsilon$ [Haberman (1983)]. The correct time variable in the saddle region after the nearly homoclinic orbit is $\tau_{+}$:

$$
\tau_{+}=t+\frac{1}{2 k} \ln \varepsilon
$$

Note that $e^{k t}=\varepsilon^{-\frac{1}{2}} e^{k \tau_{+}}$and $e^{-k t}=\varepsilon^{\frac{1}{2}} e^{-k \tau_{+}}$. When $\tau_{+}$is $O(1), e^{-k t}=O\left(\varepsilon^{\frac{1}{2}}\right)$ is small so that $y$ is near $y_{s}$ verifying that this region corresponds to being near the saddle point. Note also that $e^{k t}=O\left(\varepsilon^{-\frac{1}{2}}\right)$ is large. The two limits associated with this matching correspond to fixing $\tau_{+}$and letting $\varepsilon \rightarrow 0$, in which case from (4.1a) $t \rightarrow+\infty$, and fixing $t$ and letting $\varepsilon \rightarrow 0$, in which case from (4.1a) $\tau_{+} \rightarrow-\infty$.

The asymptotic expansion of the nearly homoclinic orbit also fails as $t \rightarrow-\infty$. In a similar way, the appropriate time variable in the saddle region before the nearly homoclinic orbit is $\tau_{-}$:

$$
\tau_{-}=t-\frac{1}{2 k} \ln \varepsilon
$$

By using the outer (nearly homoclinic) asymptotic expansion and the relationships between the inner and outer time variables $(4.1 \mathrm{a}, \mathrm{b})$, we are able to determine the form of the asymptotic expansion in the saddle region (inner expansion). For example, the term $e^{-k t}$ in the outer expansion (3.21a), yields an $O\left(\varepsilon^{\frac{1}{2}}\right)$ term in the inner expansion. 
Furthermore, it is important to notice that under the shifting of the time variable (4.1a), logarithmic terms are introduced in the following way:

$$
\begin{aligned}
t e^{-k t} & =\left(\tau_{+}-\frac{1}{2 k} \ln \varepsilon\right) \varepsilon^{\frac{1}{2}} e^{-k \tau_{+}} \\
t e^{k t} & =\left(\tau_{+}-\frac{1}{2 k} \ln \varepsilon\right) \varepsilon^{-\frac{1}{2}} e^{k \tau_{+}}
\end{aligned}
$$

Thus the terms $\varepsilon t e^{-k t}$ and $\varepsilon^{2} t e^{k t}$ yield $O\left(\varepsilon^{\frac{3}{2}} \ln \varepsilon\right)$ terms. By these observations, the method of matched asymptotic expansions implies that the asymptotic expansions in both saddle regions that surround the nearly homoclinic orbit have the same asymptotic form:

$$
y=y_{s}+\varepsilon^{\frac{1}{2}} \tilde{y}_{\frac{1}{2}}+\varepsilon \tilde{y}_{1}+\varepsilon^{\frac{3}{2}} \ln \varepsilon \tilde{y}_{*}+\varepsilon^{\frac{3}{2}} \tilde{y}_{\frac{3}{2}}+\cdots
$$

We will need the energy at successive saddle approaches. The $O\left(\varepsilon^{\frac{3}{2}}\right)$ term will not affect the energy to the order we wish to analyze. However, the $O\left(\varepsilon^{\frac{3}{2}}\right)$ term will show the weakly nonlinear interaction between the quadratic and cubic nonlinearity in the neighborhood of the saddle point. In the saddle region after the homoclinic orbit, the time variable is $\tau_{+}$, and in the region before the homoclinic orbit, the time variable is $\tau_{-}$. To avoid repetitive algebra in this paper, we use $\tau$ as the time variable in the saddle region, keeping in mind that this represents two different regions defined by (4.1a) and (4.1b). Successive saddle regions are essentially the same. Time for successive saddle approaches are shifted, and the solutions of the differential equations (the constants of integration) are different.

The leading-order terms in the differential equation in the neighborhood of the saddle point are $O\left(\varepsilon^{\frac{1}{2}}\right)$. The dissipative perturbation $-\varepsilon h$ is $O\left(\varepsilon^{\frac{3}{2}}\right)$ in the neighborhood of the saddle point, since we have assumed the dissipative perturbation is approximately linear in the neighborhood of the saddle point, $h\left(y, \frac{d y}{d t}\right) \sim \beta \frac{d y}{d t}$. The dissipation in the neighborhood of the saddle point is very important. However, if we are careful, the effect we are looking for is $O\left(\varepsilon^{\frac{3}{2}} \ln \varepsilon\right)$ and the $O\left(\varepsilon^{\frac{3}{2}}\right)$ term can be neglected. Including the $O\left(\varepsilon^{\frac{3}{2}}\right)$ terms, the system (2.1) can be written as

$$
\begin{aligned}
\varepsilon^{\frac{1}{2}} \frac{d^{2} \tilde{y}_{\frac{1}{2}}}{d \tau^{2}}+\varepsilon \frac{d^{2} \tilde{y}_{1}}{d \tau^{2}}+\varepsilon^{\frac{3}{2}} \ln \varepsilon \frac{d^{2} \tilde{y}_{*}}{d \tau^{2}}+\varepsilon^{\frac{3}{2}} \frac{d^{2} \tilde{y}_{\frac{3}{2}}}{d \tau^{2}}+\cdots \\
=\left[\varepsilon^{\frac{1}{2}} \tilde{y}_{\frac{1}{2}}+\varepsilon \tilde{y}_{1}+\varepsilon^{\frac{3}{2}} \ln \varepsilon \tilde{y}_{*}+\varepsilon^{\frac{3}{2}} \tilde{y}_{\frac{3}{2}}+\cdots\right] f_{y}\left(y_{s}\right) \\
+\varepsilon\left[\frac{1}{2}\left(\tilde{y}_{\frac{1}{2}}^{2}+2 \varepsilon^{\frac{1}{2}} \tilde{y}_{\frac{1}{2}} \tilde{y}_{1}\right) f_{y y}\left(y_{s}\right)+\cdots\right] \\
+\varepsilon^{\frac{3}{2}}\left[\frac{1}{6} \tilde{y}_{\frac{1}{2}}^{3} f_{y y y}\left(y_{s}\right)+\cdots\right]+\cdots-\varepsilon^{\frac{3}{2}} \beta \frac{d \tilde{y}_{\frac{1}{2}}}{d \tau}+\cdots
\end{aligned}
$$

In order to analyze the solution in each saddle region up to the order with which we will be concerned, we have to solve for $\tilde{y}_{\frac{1}{2}}, \tilde{y}_{1}, \tilde{y}_{*}$, and $\tilde{y}_{\frac{3}{2}}$ directly from the differential equation (4.3). For $\tilde{y}_{\frac{1}{2}}$, we consider the $O\left(\varepsilon^{\frac{1}{2}}\right)$ terms of (4.3),

$$
\frac{d^{2} \tilde{y}_{\frac{1}{2}}}{d \tau^{2}}-f_{y}\left(y_{s}\right) \tilde{y}_{\frac{1}{2}}=0
$$


This corresponds to the leading-order terms in the neighborhood of each saddle. By solving the above differential equation, $\tilde{y}_{\frac{1}{2}}$ can be represented by

$$
\tilde{y}_{\frac{1}{2}}=A e^{-k \tau}+B e^{k \tau}
$$

where $k=\sqrt{f_{y}\left(y_{s}\right)} . A$ and $B$ are the integration constants associated with a specific saddle region. These constants will be different in the saddle regions immediately before and after the nearly homoclinic orbit. The constants in the region before can be determined by matching as $\tau_{-} \rightarrow+\infty$ with the asymptotic expansion of the nearly homoclinic orbit solution as $t \rightarrow-\infty$. The constants in the region after can be determined by matching as $\tau_{+} \rightarrow-\infty$ with the asymptotic expansion of the nearly homoclinic orbit solution as $t \rightarrow+\infty$. The solution to this order in each saddle region is given exactly by (4.5), which is the same as its asymptotic expansion.

For $\tilde{y}_{1}$, we consider the $O(\varepsilon)$ terms of (4.3)

$$
\frac{d^{2} \tilde{y}_{1}}{d \tau^{2}}-k^{2} \tilde{y}_{1}=\frac{1}{2} \tilde{y}_{\frac{1}{2}}^{2} f_{y y}\left(y_{s}\right) .
$$

This term accounts for the possible quadratic nonlinearity of the force in the neighborhood of any saddle point. Using (4.5) for $\tilde{y}_{\frac{1}{2}}$, the above equation becomes

$$
\frac{d^{2} \tilde{y}_{1}}{d \tau^{2}}-k^{2} \tilde{y}_{1}=\frac{1}{2}\left(A^{2} e^{-2 k \tau}+2 A B+B^{2} e^{2 k \tau}\right) f_{y y}\left(y_{s}\right)
$$

We solve for $\tilde{y}_{1}$ in the saddle regions before and after the nearly homoclinic orbit by the method of undetermined coefficients:

$$
\tilde{y}_{1}=\frac{1}{6 k^{2}} f_{y y}\left(y_{s}\right)\left[A^{2} e^{-2 k \tau}-6 A B+B^{2} e^{2 k \tau}\right] .
$$

The method of matched asymptotic expansions implies that there are no homogeneous solutions $e^{ \pm k \tau}$ at this order because, for example, there are no terms of $O\left(\varepsilon^{3 / 2} e^{k t}\right)$ or $O\left(\varepsilon^{5 / 2} e^{-k t}\right)$ in (3.21a). A similar observation is necessary in (3.21b).

The next order, $O\left(\varepsilon^{3 / 2} \ln \varepsilon\right)$, solution $\tilde{y}_{*}$ satisfies the following linear homogeneous differential equation:

$$
\frac{d^{2} \tilde{y}_{*}}{d \tau^{2}}-k^{2} \tilde{y}_{*}=0
$$

Similar to the calculation for $\tilde{y}_{\frac{1}{2}}, \tilde{y}_{*}$ can be represented by

$$
\tilde{y}_{*}=A_{*} e^{-k \tau}+B_{*} e^{k \tau} \text {. }
$$

At order $O\left(\varepsilon^{\frac{3}{2}}\right)$, the solution $\tilde{y}_{\frac{3}{2}}$ satisfies the following linear nonhomogeneous differential equation corresponding to the interaction of the quadratic and cubic nonlinearities:

$$
\begin{aligned}
\frac{d^{2} \tilde{y}_{\frac{3}{2}}}{d \tau^{2}}-k^{2} \tilde{y}_{\frac{3}{2}}= & \tilde{y}_{\frac{1}{2}} \tilde{y}_{1} f_{y y}\left(y_{s}\right)+\frac{1}{6} \tilde{y}_{\frac{1}{2}}^{3} f_{y y y}\left(y_{s}\right)-\beta \frac{d \tilde{y}_{\frac{1}{2}}}{d \tau} \\
= & \frac{\sigma}{2}\left(A^{2} B e^{-k \tau}+A B^{2} e^{k \tau}\right)+k \beta\left(A e^{-k \tau}-B e^{k \tau}\right) \\
& +O\left(e^{3 k \tau}, e^{-3 k \tau}\right)
\end{aligned}
$$


where $\sigma$ is defined by (3.10) and $O\left(e^{3 k \tau}, e^{-3 k \tau}\right)$ represents terms we can calculate but do not need. Exponentially resonant terms contribute to the solution

$$
\begin{array}{r}
\tilde{y}_{\frac{3}{2}}=A_{\frac{3}{2}} e^{-k \tau}+B_{\frac{3}{2}} e^{k \tau}-\left(\frac{\sigma}{4 k} A^{2} B+\frac{\beta}{2} A\right) \tau e^{-k \tau} \\
+\left(\frac{\sigma}{4 k} A B^{2}-\frac{\beta}{2} B\right) \tau e^{k \tau}+O\left(e^{3 k \tau}, e^{-3 k \tau}\right)
\end{array}
$$

In conclusion, the expansion of each saddle solution (up to the order in which we are interested) is

$$
\begin{aligned}
y=y_{s}+ & \varepsilon^{\frac{1}{2}}\left(A e^{-k \tau}+B e^{k \tau}\right)+\varepsilon \frac{1}{6 k^{2}} f_{y y}\left(y_{s}\right)\left[A^{2} e^{-2 k \tau}-6 A B+B^{2} e^{2 k \tau}\right] \\
& +\varepsilon^{\frac{3}{2}} \ln \varepsilon\left(A_{*} e^{-k \tau}+B_{*} e^{k \tau}\right) \\
& +\varepsilon^{\frac{3}{2}}\left[A_{\frac{3}{2}} e^{-k \tau}+B_{\frac{3}{2}} e^{k \tau}-\left(\frac{\sigma}{4 k} A^{2} B+\frac{\beta}{2} A\right) \tau e^{-k \tau}\right. \\
& \left.+\left(\frac{\sigma}{4 k} A B^{2}-\frac{\beta}{2} B\right) \tau e^{k \tau}+O\left(e^{3 k \tau}, e^{-3 k \tau}\right)\right]+\cdots
\end{aligned}
$$

The constants in each saddle region can be determined by matching ( $\tau \rightarrow \pm \infty$ and $t \rightarrow \pm \infty)$ to the nearly homoclinic orbit.

\section{Matching between the saddle region and the nearly homoclinic orbit}

In order to find the change in the energy from one saddle approach to the next, we match the asymptotic solutions in both saddle regions to the nearly homoclinic orbit between them. As $t \rightarrow-\infty\left(\tau_{-} \rightarrow \infty\right)$, the nearly homoclinic orbit approaches the preceding saddle region, and as $t \rightarrow \infty\left(\tau_{+} \rightarrow-\infty\right)$, the nearly homoclinic orbit approaches the following saddle region. According to (3.21a) and (3.21b), the corresponding asymptotic expansions for the saddle approach prior to the nearly homoclinic orbit $(t \rightarrow-\infty)$ and for the saddle approach after the nearly homoclinic orbit $(t \rightarrow \infty)$ can be determined by matching to the saddle solution (4.12) as $\tau_{-} \rightarrow \infty$ and $\tau_{+} \rightarrow-\infty$.

As $t \rightarrow \infty$ (equivalent by matching to $\tau_{+} \rightarrow-\infty$ ), the asymptotic expansion of the nearly homoclinic orbit (3.21a) expressed in terms of the saddle variables is

$$
\begin{aligned}
y \sim y_{s} & +\varepsilon^{\frac{1}{2}} \alpha_{0}\left[1+\varepsilon \ln \varepsilon\left(\frac{\beta}{4 k}-\frac{Q \sigma}{16 k^{4}}\right)\right] e^{-k \tau_{+}} \\
& -\varepsilon^{\frac{1}{2}} \frac{Q}{2 k^{2} \alpha_{0}}\left[1+\varepsilon \ln \varepsilon\left(\frac{\beta}{4 k}+\frac{Q \sigma}{16 k^{4}}\right)\right] e^{k \tau_{+}}+\cdots
\end{aligned}
$$

By matched asymptotic expansions, (5.1) should be the same as the asymptotic expansion of the corresponding saddle solution (4.12). By comparing this to the solution in the saddle region after the nearly homoclinic orbit, we determine the constants of 
that saddle region:

$$
\begin{aligned}
A & =\alpha_{0} \\
B & =-\frac{Q}{2 k^{2} \alpha_{0}} \\
A_{*} & =\frac{\alpha_{0} \beta}{4 k}-\frac{Q \sigma \alpha_{0}}{16 k^{4}} \\
B_{*} & =-\frac{Q \beta}{8 k^{3} \alpha_{0}}-\frac{Q^{2} \sigma}{32 k^{6} \alpha_{0}}
\end{aligned}
$$

We can determine these coefficients by comparison with the homoclinic expansion (3.21a) if we note that $t \Rightarrow-\frac{1}{2 k} \ln \varepsilon$.

In the saddle region before the nearly homoclinic orbit, the time variable $\tau_{-}$is simply the nearly homoclinic time variable $\mathrm{t}$ shifted backwards by $-\frac{1}{2 k} \ln \varepsilon, \tau_{-}=$ $t-\frac{1}{2 k} \ln \varepsilon$. It is important to notice that under this shifting of the time variable, logarithmic terms are introduced in the following way :

$$
\begin{aligned}
t e^{-k t} & =\left(\tau_{-}+\frac{1}{2 k} \ln \varepsilon\right) \varepsilon^{-\frac{1}{2}} e^{-k \tau_{-}}, \\
t e^{k t} & =\left(\tau_{-}+\frac{1}{2 k} \ln \varepsilon\right) \varepsilon^{\frac{1}{2}} e^{k \tau_{-}} .
\end{aligned}
$$

Thus, as $t \rightarrow-\infty\left(\tau_{-} \rightarrow+\infty\right)$, the asymptotic expansion of the nearly homoclinic orbit (3.21b) has the following asymptotic expansion in terms of the saddle variables:

$$
\begin{aligned}
y \sim y_{s} & +\varepsilon^{\frac{1}{2}} \alpha_{0}\left[1+\varepsilon \ln \varepsilon\left(\frac{(Q+M) \sigma}{16 k^{4}}-\frac{\beta}{4 k}\right)\right] e^{k \tau_{-}} \\
& -\frac{\varepsilon^{\frac{1}{2}}}{2 k^{2} \alpha_{0}}\left[(Q+M)+\varepsilon \ln \varepsilon\left(-\frac{(Q+M) \beta}{4 k}-\frac{(Q+M)^{2} \sigma}{16 k^{4}}\right)\right] e^{-k \tau_{-}}+\cdots
\end{aligned}
$$

By matched asymptotic expansions, (5.4) should be the same as the asymptotic expansion of the saddle solution preceding the nearly homoclinic orbit. By comparing this to the solution in the saddle region before the nearly homoclinic orbit, we determine the constants of that saddle region:

$$
\begin{aligned}
B & =\alpha_{0}, \\
A & =-\frac{Q+M}{2 k^{2} \alpha_{0}}, \\
-B_{*} & =\frac{\alpha_{0} \beta}{4 k}-\frac{(Q+M) \sigma \alpha_{0}}{16 k^{4}}, \\
-A_{*} & =-\frac{(Q+M) \beta}{8 k^{3} \alpha_{0}}-\frac{(Q+M)^{2} \sigma}{32 k^{6} \alpha_{0}} .
\end{aligned}
$$

By matching, we have shown that the parameters in two consecutive saddle regions are related by (5.2) and (5.5).

\section{Change of the energy between two consecutive saddle approaches}

The preceding sections showed a detailed calculation of a nearly homoclinic orbit and the saddle approaches that surround it. We now show the relationship between the 
time dependent solution and its energy. The formula for the energy in saddle regions is unchanged,

$$
H=\frac{1}{2}\left(\frac{d y}{d t}\right)^{2}-\int_{y_{S}}^{y} f(\hat{y}) d \hat{y}=\frac{1}{2}\left(\frac{d y}{d \tau}\right)^{2}-\int_{y_{S}}^{y} f(\hat{y}) d \hat{y}
$$

since in the saddle region time is shifted and therefore $d t=d \tau$.

We now find an approximate expression for the energy in any saddle region. Using the Taylor expansion of $f(y)$ and noting that at the saddle $f\left(y_{s}\right)=0$, yields

$$
H=\frac{1}{2}\left(\frac{d y}{d \tau}\right)^{2}-\left[\frac{1}{2} f_{y}\left(y_{s}\right)\left(y-y_{s}\right)^{2}+\frac{1}{6} f_{y y}\left(y_{s}\right)\left(y-y_{s}\right)^{3}+O\left[\left(y-y_{s}\right)^{4}\right]\right]
$$

Since (4.12) represents the solution in any saddle region including the $O\left(\varepsilon^{\frac{3}{2}}\right)$ terms, by substituting (4.12) into (6.2) we will obtain the asymptotic expansion of the energy in the saddle region,

$$
H=-2 k^{2}\left[\varepsilon A B+\varepsilon^{2} \ln \varepsilon\left(A B_{*}+B A_{*}\right)\right]+\cdots .
$$

Since (6.3) is valid in the entire saddle region, we conclude that the energy is nearly constant (up to this order) everywhere in each saddle region. Time-dependence in the expression of $\mathrm{H}$ occurs only in those terms of $O\left(\varepsilon^{2}\right)$ or higher. Equation (6.3) is very convenient to compare the difference in the energy between two consecutive saddle approaches up to $O\left(\varepsilon^{2} \ln \varepsilon\right)$, because the result will be the same if we compare any two points in the corresponding saddle regions. As shown in the next section, this conclusion is particularly convenient when we compare the numerical solution to this theory.

From (5.5) and (6.3), the energy at the saddle region $\left(H_{-}\right)$before the nearly homoclinic orbit has the asymptotic expansion

$$
H_{-}=\varepsilon(Q+M)+\varepsilon^{2} \ln \varepsilon \frac{-\beta(Q+M)}{2 k}+\cdots .
$$

At the next saddle region immediately after the nearly homoclinic orbit, the energy $\left(H_{+}\right)$is determined from (5.2) and (6.3):

$$
H_{+}=\varepsilon Q+\varepsilon^{2} \ln \varepsilon \frac{\beta Q}{2 k}+\cdots
$$

By comparing the energies at two consecutive saddle regions, (6.4) and (6.5), we determine the change in the energy from one saddle approach to the next:

$$
\Delta H \equiv H_{+}-H_{-} \sim-\varepsilon M+\varepsilon^{2} \ln \varepsilon\left(\frac{\beta}{k}\right)\left(Q+\frac{M}{2}\right)+\cdots
$$

To leading order, it is well-known that the change in the energy over a nearly homoclinic orbit is $-\varepsilon M$, where $M$ is the usual Melnikov integral

$$
M=2 \int_{0}^{\infty} h\left(y_{0}, y_{0 t}\right) y_{0} d t=\oint h\left(y_{0}, \pm \sqrt{-2 V\left(y_{0}\right)}\right) d y_{0}
$$

The $O\left(\varepsilon^{2} \ln \varepsilon\right)$ terms in (6.6) represent an $O(\varepsilon \ln \varepsilon)$ percentage correction to the wellknown leading-order change of the energy. According to (6.6), this $O\left(\varepsilon^{2} \ln \varepsilon\right)$ correction 
depends on $Q$ where, according to (6.5), $\varepsilon Q$ is the leading-order energy at the saddle region after the nearly homoclinic orbit,

$$
\Delta H \sim-\varepsilon M+\varepsilon^{2} \ln \varepsilon\left(\frac{\beta}{k}\right)\left(\frac{H_{+}}{\varepsilon}+\frac{M}{2}\right)+\cdots .
$$

In fact, the $O\left(\varepsilon^{2} \ln \varepsilon\right)$ correction is proportional to $Q+\frac{M}{2}$ which is the average of the leading-order energies at the saddle regions before and after the nearly homoclinic orbit,

$$
\Delta H \sim-\varepsilon M+\varepsilon^{2} \ln \varepsilon\left(\frac{\beta}{k}\right)\left(\frac{H_{+}+H_{-}}{2 \varepsilon}\right)+\cdots .
$$

Furthermore, the correction to the change in the energy is also proportional to the ratio of the linear damping coefficient of the perturbation at the saddle point of the unperturbed system $\left(\beta=h_{v}\left(y_{s}, 0\right)\right)$ to the linear exponential growth and decay rate $(k)$ at the saddle point. Even though the detailed calculations of the nearly homoclinic orbit require the nonlinear effect $(\sigma)$ of the saddle point, the $O\left(\varepsilon^{2} \ln \varepsilon\right)$ correction to the energy change does not depend on the higher-order nonlinearities of the potential function at the saddle point. If the damping is not linear around the saddle point (with nonlinearities smaller than the size of linear terms), then $\beta=0$ in this analysis and the $O\left(\varepsilon^{2} \ln \varepsilon\right)$ terms vanish, in which case correction to the leading-order dissipation of the energy will be smaller than $O\left(\varepsilon^{2} \ln \varepsilon\right)$. (If the damping is locally larger than linear, then the effect will be larger than $O\left(\varepsilon^{2} \ln \varepsilon\right)$ ). It can be seen that these $O\left(\varepsilon^{2} \ln \varepsilon\right)$ corrections are due to the $t e^{ \pm k t}$ terms in (3.21) with the amplitude factor $t$ replaced by $\pm \frac{1}{2 k} \ln \varepsilon$, depending on whether the saddle approach occurs before or after the nearly homoclinic orbit.

It is well-known that the leading-order change of the energy along a nearly homoclinic orbit is a constant, the Melnikov integral. However, the $O\left(\varepsilon^{2} \ln \varepsilon\right)$ correction depends on the energy at the saddle region. Thus, if we consider a sequence of nearly homoclinic orbits, then this correction is different for each nearly homoclinic orbit. This recursive effect has been analyzed in Haberman and Ho (1995b), where a calculation was performed on the boundary of the basin of attraction which requires a sequence of nearly homoclinic orbits.

\section{Numerical comparison for a specific double-well potential}

As a simple illustration, the one-dimensional motion of a particle in a specific doublewell potential is considered:

$$
\frac{d^{2} y}{d t^{2}}+\left(y^{3}-y^{2}-2 y\right)=-\varepsilon 2 \frac{d y}{d t}
$$

with a small purely dissipative perturbation, $-2 \varepsilon \frac{d y}{d t}$. The system consists of two competing equilibria (left and right) and one saddle point in between at $y_{s}=0$. The energy $H$ is

$$
H=\frac{1}{2}\left(\frac{d y}{d t}\right)^{2}+V(y)
$$

where $V(y)=\frac{1}{4} y^{4}-\frac{1}{3} y^{3}-y^{2}$ is the potential energy and is not symmetric (around $y=0$ ). 
In the last section, the change of the energy between two consecutive saddle approaches for the left nearly homoclinic orbit is given by (6.6):

$$
\Delta H=-\varepsilon M_{L}+\varepsilon^{2} \ln \varepsilon \frac{\beta}{k}\left(\frac{H_{0}}{\varepsilon}+\frac{1}{2} M_{L}\right),
$$

where $M_{L}$ stands for the Melnikov integral for the left homoclinic orbit and $H_{0}$ is the energy at the preceding saddle. We are interested in comparing this to the change in the energy between saddle approaches produced by numerically integrating the time-dependent differential equation, (7.1). Here, we verify (7.3) including the coefficient of the $O\left(\varepsilon^{2} \ln \varepsilon\right)$ term by computing numerically the change in the energy. For this particular example, $\beta=2, k=\sqrt{2}, M_{L}=3.565721510024 \ldots$, and $M_{R}=16.72981910456 \ldots$. With these values of the parameters, the coefficient of the $O\left(\varepsilon^{2} \ln \varepsilon\right)$ for the change of the energy can be determined with the given energy $H_{0}$ at the preceding saddle approach. Since we are considering the nearly homoclinic orbit, $H_{0}$ must be $O(\varepsilon)$. Furthermore, since the leading-order change of the energy is negative, in order to compare two consecutive saddle approaches before the trajectory gets captured, the initial energy must be at least $\varepsilon\left(M_{R}+M_{L}\right)$. With this constraint in mind, we choose the initial condition to be $H_{0}=22 \varepsilon$, so that the asymptotic formula for the higher-order change of the energy, $\Delta H^{(1)}$, from one saddle approach to the next is

$$
\Delta H^{(1)}=\Delta H+\varepsilon M_{L}=33.6341 \varepsilon^{2} \ln \varepsilon+\cdots
$$

The correction to the change of the energy theoretically contains not just the $O\left(\varepsilon^{2} \ln \varepsilon\right)$ term but also higher-order terms. A term of $O\left(\varepsilon^{2}\right)$ should be included in a numerical comparison because it is not much smaller than the $O\left(\varepsilon^{2} \ln \varepsilon\right)$ term:

$$
\Delta H^{(1)} \equiv \Delta H+\varepsilon M_{L}=C_{*} \varepsilon^{2} \ln \varepsilon+C_{2} \varepsilon^{2}+\cdots
$$

where $C_{*}$ is the coefficient we want to compare with the analytical result. To find $C_{*}$ (and $C_{2}$ ), we use Richardson extrapolation. We evaluate $\Delta H^{1}(\varepsilon)$ with two different values of $\varepsilon$ and approximate the coefficient of the $O\left(\varepsilon^{2} \ln \varepsilon\right)$ term by using (7.5). With two different $\varepsilon, \varepsilon_{1}$ and $\varepsilon_{1} / 2, C_{*}$ can be expressed as

$$
C_{*}=\frac{1}{\left(\varepsilon_{1}\right)^{2} \ln (2)}\left[\Delta H^{(1)}\left(\varepsilon_{1}\right)-4 \Delta H^{(1)}\left(\frac{\varepsilon_{1}}{2}\right)\right]+\cdots .
$$

In Table 1, numerical data is given for a left nearly homoclinic orbit with energy at the preceding saddle, $H_{0}=22 \varepsilon$. According to Table 1 , when $\varepsilon$ decreases from $10^{-4}$ to $10^{-6}$, the numerical approximations of $C_{*}$ converge to the analytical result $33.6341 \ldots$ Moreover, the numerical approximation of $C_{*}=33.6174 \ldots$ in the 6 th row is the closest approximation. The small difference between the analytical and numerical result, $\Delta C_{*} \approx 1.7 * 10^{-2}$, is caused by higher-order terms in (7.5). The percent error for $C_{*}$ is very small, showing the accuracy of the analytical result (7.3) for the saddle-to-saddle change of the energy. We note that the numerical results reported in Table 1 are not reliable when $\varepsilon$ is too small (probably due to the round-off error of $O\left(10^{-14}\right)$ in the numerical integration over one complete homoclinic orbit), in which case the analytical formula may (of course) still be used. 


\begin{tabular}{cccccc}
\hline \hline$\varepsilon_{1}$ & $\varepsilon_{1} / 2$ & $\Delta H^{(1)}\left(\varepsilon_{1}\right)$ & $\Delta H^{(1)}\left(\varepsilon_{1} / 2\right)$ & $C_{*}$ & $C_{2}$ \\
\hline $1.0000 \mathrm{e}-4$ & $5.0000 \mathrm{e}-5$ & $-3.72521 \mathrm{e}-6$ & $-9.89929 \mathrm{e}-7$ & $3.38324 \mathrm{e}+1$ & $-6.09129 \mathrm{e}+1$ \\
$5.0000 \mathrm{e}-5$ & $2.5000 \mathrm{e}-5$ & $-9.89929 \mathrm{e}-7$ & $-2.62120 \mathrm{e}-7$ & $3.37883 \mathrm{e}+1$ & $-6.13491 \mathrm{e}+1$ \\
$2.5000 \mathrm{e}-5$ & $1.2500 \mathrm{e}-5$ & $-2.62120 \mathrm{e}-7$ & $-6.91854 \mathrm{e}-8$ & $3.37517 \mathrm{e}+1$ & $-6.17375 \mathrm{e}+1$ \\
$1.2500 \mathrm{e}-5$ & $6.2500 \mathrm{e}-6$ & $-6.91854 \mathrm{e}-8$ & $-1.82094 \mathrm{e}-8$ & $3.37210 \mathrm{e}+1$ & $-6.20845 \mathrm{e}+1$ \\
$6.2500 \mathrm{e}-6$ & $3.1250 \mathrm{e}-6$ & $-1.82094 \mathrm{e}-8$ & $-4.78044 \mathrm{e}-9$ & $3.36974 \mathrm{e}+1$ & $-6.23665 \mathrm{e}+1$ \\
\hline $3.1250 \mathrm{e}-6$ & $1.5625 \mathrm{e}-6$ & $-4.78044 \mathrm{e}-9$ & $-1.25209 \mathrm{e}-9$ & $3.36174 \mathrm{e}+1$ & $-6.27426 \mathrm{e}+1$ \\
\hline $1.5625 \mathrm{e}-6$ & $7.8125 \mathrm{e}-7$ & $-1.25209 \mathrm{e}-9$ & $-3.27244 \mathrm{e}-10$ & $3.33680 \mathrm{e}+1$ & $-6.34160 \mathrm{e}+1$ \\
$7.8125 \mathrm{e}-7$ & $3.9063 \mathrm{e}-7$ & $-3.27244 \mathrm{e}-10$ & $-8.53401 \mathrm{e}-11$ & $3.24259 \mathrm{e}+1$ & $-6.69226 \mathrm{e}+1$ \\
$3.9063 \mathrm{e}-7$ & $1.9531 \mathrm{e}-7$ & $-8.53401 \mathrm{e}-11$ & $-2.21924 \mathrm{e}-11$ & $3.49303 \mathrm{e}+1$ & $-8.08243 \mathrm{e}+1$ \\
\hline
\end{tabular}

TABLE 1. Coefficient of $O\left(\varepsilon^{2} \ln \varepsilon\right)$ term for the change of the energy $\left(H_{0}=22 \varepsilon\right)$.

Here, $H_{0}$ is the initial energy, $\varepsilon_{1}$ and $\varepsilon_{1} / 2$ are the two epsilons used in the Richardson extrapolation, $\Delta H^{(1)}\left(\varepsilon_{1}\right)$ and $\Delta H^{(1)}\left(\varepsilon_{1} / 2\right)$ are the numerical results of the higherorder change of the energy corresponding to $\varepsilon_{1}$ and $\varepsilon_{1} / 2, C_{*}$ and $C_{2}$ are the coefficients of the $O\left(\varepsilon^{2} \ln \varepsilon\right)$ and the $O\left(\varepsilon^{2}\right)$ terms predicted by Richardson extrapolation.

\section{Appendix. Asymptotic expansion of the second homogeneous solution}

In this appendix, we derive the second homogeneous solution $y_{H}$ for the following differential equation arising from analyzing nearly homoclinic orbits:

$$
\frac{d^{2} y_{H}}{d t^{2}}-f_{y}\left(y_{0}\right) y_{H}=0
$$

where $y_{0}$ is a homoclinic orbit which we have defined to be an even function of $\mathrm{t}$ (around $t=0)$. Furthermore, we also derive the asymptotic behavior of $y_{H}$ as $t \rightarrow+\infty$. Due to the translation invariance of (3.3), one of the homogeneous solutions of (A.1) can be defined as $y_{0 t}$, which is an odd function of $t$. The second homogeneous solution can be defined to be the unique even solution of the Wronskian relation:

$$
y_{0} y_{H t}-y_{0 t} y_{H}=1 \text {. }
$$

We can derive an explicit formula for $y_{H}$ (without singular integrals),

$$
y_{H}(t)=y_{0} \int_{0}^{t}\left[\left(y_{0}\right)^{-2}-f\left(y^{*}\right)^{-2} t^{-2}\right] d t-\frac{f\left(y^{*}\right)^{-2}}{t} y_{0},
$$

where $y^{*}=y_{0}(0)$ is the maximum value of $y$ associated with the homoclinic orbit. However, we will use (A.2) instead to obtain the needed asymptotic behavior of $y_{H}$ as $t \rightarrow+\infty$.

Since $y_{H}$ is a solution of (A.1) and $f_{y}\left(y_{0}\right) \sim k^{2}$ as $t \rightarrow \infty, y_{H}$ satisfies to leading order as $t \rightarrow+\infty$,

$$
\frac{d^{2} y_{H}}{d t^{2}}-\left(k^{2}+\cdots\right) y_{H}=0
$$


Since $y_{0}$ exponentially decays, from (A.2) $y_{H}$ exponentially grows as $t \rightarrow+\infty$

$$
y_{H} \sim E e^{k t}+g(t)+\cdots, \quad \text { as } t \rightarrow+\infty,
$$

where we are particularly interested in obtaining higher-order terms than just the simple growing exponential. By substituting the expansions of $y_{H}$ and $y_{0}$ from (A.5) and (3.7), respectively, into the Wronskian relationship (A.2), we obtain

$$
\begin{aligned}
& -2 \alpha_{0} k^{2} E-\frac{\alpha_{0}^{2} f_{y y}\left(y_{s}\right) E}{3} e^{-k t}-\alpha_{0} k g^{\prime} e^{-k t} \\
& \quad-\frac{2 \alpha_{0}^{2} f_{y y}\left(y_{s}\right) E}{3} e^{-k t}-g(t) \alpha_{0} k^{2} e^{-k t}+\cdots=1 .
\end{aligned}
$$

By considering the $O(1)$ terms of (A.6), it follows that $E=-1 / 2 \alpha_{0} k^{2}$.

The largest term that remains in (A.6) is $O\left(e^{-k t}\right)$ which must be balanced by the $\left(g^{\prime}+k g\right) e^{-k t}$ terms. Thus $g^{\prime}+k g=O(1)$ or $g(t)=O(1)$, ignoring the exponentially small homogeneous solutions. From (A.6), we obtain

$$
g(t)=\frac{f_{y y}\left(y_{s}\right)}{2 k^{4}}
$$

Therefore, the asymptotic expansion of $y_{H}$ as $t \rightarrow \infty$ is of the form

$$
y_{H} \sim \frac{-1}{2 \alpha_{0} k^{2}}\left[e^{k t}-\frac{\alpha_{0} f_{y y}\left(y_{s}\right)}{k^{2}}+R\right]
$$

where $R$ represents the next term.

However, if $y_{H}$ has a term of $O\left(t e^{-k t}\right)$, it will contribute in the matching due to the logarithmic time shift from the center to the saddle point. Consequently, a higher-order calculation of $y_{H}$ is needed. It is simpler to determine the next term by considering the differential equation for $y_{H}$ directly near the saddle point. By using (3.5b), the asymptotic expansion of $y_{0},(\mathrm{~A} .1)$ becomes

$$
\begin{gathered}
y_{H t t}-\left\{k^{2}+\left(\alpha_{0} e^{-k t}+\frac{\alpha_{0}^{2} f_{y y}\left(y_{s}\right)}{6 k^{2}} e^{-2 k t}\right) f_{y y}\left(y_{s}\right)\right. \\
\left.+\frac{1}{2} \alpha_{0}^{2} e^{-2 k t} f_{y y y}\left(y_{s}\right)+\cdots\right\} y_{H}=0 .
\end{gathered}
$$

Since the differential equation (A.8) for $y_{H}$ is linear, it is particularly easy to obtain the next term $R$. By substituting expansion (A.7) into (A.8), we obtain

$$
\begin{aligned}
& \frac{-1}{2 \alpha_{0} k^{2}}\left(k^{2} e^{k t}+R_{t t}\right)-\left\{k^{2}+\alpha_{0} e^{-k t} f_{y y}\left(y_{s}\right)+\frac{\alpha_{0}^{2}}{2}\left[f_{y y y}\left(y_{s}\right)+\frac{f_{y y}^{2}\left(y_{s}\right)}{3 k^{2}}\right] e^{-2 k t}\right\} \\
& \quad \times \frac{-1}{2 \alpha_{0} k^{2}}\left(e^{k t}-\frac{\alpha_{0} f_{y y}\left(y_{s}\right)}{k^{2}}+R\right)=0 .
\end{aligned}
$$

By considering the most important exponentially small terms, $R$ satisfies the following differential equation:

$$
R_{t t}-k^{2} R=\frac{\alpha_{0}^{2}}{2}\left[f_{y y y}\left(y_{s}\right)-\frac{5 f_{y y}^{2}\left(y_{s}\right)}{3 k^{2}}\right] e^{-k t}
$$


Since $e^{-k t}$ is a homogeneous solution of the above differential equation, the particular solution will be $O\left(t e^{-k t}\right)$. By using the method of undetermined coefficients, the particular solution is

$$
R=\frac{-\alpha_{0}^{2}}{4 k}\left(f_{y y y}\left(y_{s}\right)-\frac{5 f_{y y}^{2}\left(y_{s}\right)}{3 k^{2}}\right) t e^{-k t} .
$$

Since we assume $R$ is the next higher-order term in the expansion (A.7), we can ignore the homogeneous solution of $\mathrm{R}$ which involves $O\left(e^{k t}\right)$. The $O\left(e^{-k t}\right)$ homogeneous solutions are asymptotically smaller than (A.10). Moreover, this $O\left(t e^{-k t}\right)$ term will contribute to the logarithmic terms in the saddle solution because of the logarithmic time shift. By combining (A.10) and (A.7), we derive that $y_{H}$ has an asymptotic expansion

$$
y_{H} \sim \frac{-1}{2 \alpha_{0} k^{2}}\left[e^{k t}-\frac{\alpha_{0} f_{y y}\left(y_{s}\right)}{k^{2}}-\frac{\alpha_{0}^{2}}{4 k} \sigma t e^{-k t}\right], \quad \text { as } t \rightarrow+\infty,
$$

where $\sigma=f_{y y y}\left(y_{s}\right)-5 f_{y y}^{2}\left(y_{s}\right) / 3 k^{2}$, which was important in the asymptotic analysis in this paper.

\section{References}

1. F. J. Bourland and R. Haberman, Separatrix crossing: time-invariant potentials with dissipation, SIAM J. Appl. Math. 50 (1990), 1716-1744.

2. _ Capture and the connection formulas for the transition across a separatrix, In: Asymptotic Analysis and the Numerical Solution of Partial Differential Equations, (H. G. Kaper and M. Garbey, eds.), Dekker, New York, 1991, pp. 17-30.

3. _ Connection across a separatrix with dissipation, Studies in Appl. Math. 91 (1994), 95124.

4. F. J. Bourland, R. Haberman, and W. L. Kath, Averaging methods for the phase shift of arbitrarily perturbed strongly nonlinear oscillators with an application to capture, SIAM J. Appl. Math. 51 (1991), 1150-1167.

5. J. Guckenheimer and P. Holmes, Nonlinear Oscillations, Dynamical Systems, and Bifurcations of Vector Fields, Springer-Verlag, New York, 1983.

6. R. Haberman, Energy bounds for the slow capture by a center in sustained resonance, SIAM J. Appl. Math. 43 (1983), 244-256.

7. R. Haberman and E. K. Ho, Boundary of the basin of attraction for weakly damped primary resonance, ASME J. Appl. Mech., accepted, 1995a.

8. L Logarithmic correction to the probability of capture for dissipatively perturbed Hamiltonian systems, Chaos, accepted, 1995b.

9. J. Henrard, The adiabatic invariant in classical mechanics, In: Dynamics Reported: Expositions in Dynamical Systems, (C. K. R. T. Jones, U. Kirchgraber, and W. O. Waither, eds.), SpringerVerlag, Berlin, 1993.

10. E. K. Ho, Hamiltonian Systems with Dissipation: Basins of Attraction and Nearly Homoclinic Orbits, Ph. D. thesis, Southern Methodist University, 1993.

11. J. Kevorkian and J. D. Cole, Perturbation Methods in Applied Mathematics, Springer-Verlag, New York, 1981.

12. C. G. Lange, On spurious solutions of singular perturbation problems, Studies in Appl. Math. 68 (1983), 227-257.

13. A. I. Neishtadt, Change of an adiabatic invariant at a separatrix, Sov. J. Plasma Phys. 12 (1986), $568-573$. 
14. _ Probability phenomena due to separatrix crossing, Chaos 1 (1991), 42-48.

15. J. L. Tennyson, J. R. Cary, and D. F. Escande, Change of the adiabatic invariant due to separatrix crossing, Phys. Rev. Lett. 56 (1986), 2117-2120.

16. A. V. Timofeev, On the constancy of an adiabatic invariant when the nature of the motion changes, Zh. Eksp. Teor. Fiz. 75 (1978), 1303-1308. (In Russian.) Sov. Phys. JETP 48 (1978), 656-659.

17. S. Wiggins, Introduction to Applied Nonlinear Dynamical Systems and Chaos, Springer-Verlag, New York 1990.

Department of Mathematics, Southern Methodist University, Dallas, Texas 75275, U.S.A. 